\title{
The Locus of Motor Activity in the Superior Colliculus of the Rhesus Monkey Is Unaltered during Saccadic Adaptation
}

\author{
Stephan Quessy, ${ }^{1}$ Julie Quinet, ${ }^{4}$ and Edward G. Freedman ${ }^{1,2,3}$ \\ Departments of ${ }^{1}$ Neurobiology and Anatomy and ${ }^{2}$ Biomedical Engineering, and ${ }^{3}$ Center for Visual Science, University of Rochester, Rochester, New York \\ 14642, and ${ }^{4}$ Department of Otolaryngology and Communicative Sciences, University of Mississippi Medical Center, Jackson, Mississippi 39216
}

The location of motor-related activity in the deeper layers of the superior colliculus (SC) is thought to generate a desired displacement command specifying the amplitude and direction of saccadic eye movements. However, the amplitude of saccadic eye movements made to visual targets can be systematically altered by surreptitiously moving the target location after the saccade has been initiated. Depending on whether the target is moved closer to or further from the fixation location, adaptation of saccade amplitude results in movements that are either smaller or larger than control movements. It remains an open question whether the SC specifies the desired movement to the original target location or whether SC activity specifies the vector of the amplitude-altered movement that is observed as adaptation progresses. We investigated this question by recording the activity of saccade-related burst neurons in the SC of head-restrained rhesus monkeys during both backward and forward saccadic adaptation. During adaptation in each direction, we find no evidence that is consistent with a change in the locus of SC activity despite changes in saccade amplitude; the location of SC motor-related activity does not appear to be remapped during either forward or backward saccadic adaptation. These data are inconsistent with hypotheses that propose a key role for the SC in mediating the changes in saccade amplitude observed during adaptation.

\section{Introduction}

Movements planned and executed through the concerted actions of neuromuscular systems do not always produce the intended results. If errors are persistent and systematic, changes in the neural command signals may attempt to adjust the output to reduce or eliminate discrepancies between desired and actual movements. Consideration of these adaptive changes is important if we are to understand the self-correcting mechanisms used by the brain in an attempt to maintain the accuracy of goaldirected behaviors.

Adaptation of the amplitude of saccadic eye movements has often been investigated by introducing a visual error at the end of saccades (McLaughlin, 1967; Miller et al., 1981; Deubel et al., 1986; Frens and van Opstal, 1994; Frens and Van Opstal, 1997; Phillips et al., 1997; Straube et al., 1997; Scudder et al., 1998; Wallman and Fuchs, 1998; Noto et al., 1999; Hopp and Fuchs, 2002, 2006; Robinson et al., 2003; Alahyane and Pélisson, 2004, 2005; Takeichi et al., 2005, 2007; Cecala and Freedman, 2008, 2009; Ethier et al., 2008). Over the course of repeated trials the amplitude of the initial saccade changes so that the error at the end of the primary saccade is reduced or increased depending on the direction of introduced visual error (McLaughlin, 1967; Straube et al., 1997; Noto et al., 1999; Hopp and Fuchs, 2004).

\footnotetext{
Received June 17, 2010; revised Aug. 2, 2010; accepted Aug. 30, 2010.

This work was supported in part by National Institutes of Health Grant EY13239 (E.G.F.). We thank G. Parker for technical assistance, and Dr.M. M. G. Walton and Adam Pallus for comments on a previous version of the manuscript. Correspondence should be addressed to Edward G. Freedman, Departments of Neurobiology and Anatomy and Biomedical Engineering, and Center for Visual Science, University of Rochester, Box 603, Rochester, NY 14642. E-mail: ed_freedman@urmc.rochester.edu.

DOI:10.1523/JNEUROSCI.3111-10.2010

Copyright $\odot 2010$ the authors $\quad 0270-6474 / 10 / 3014235-10 \$ 15.00 / 0$
}

The locus of motor-related activity in the superior colliculus (SC) specifies the amplitude and direction of changes in the direction of the line of sight (Wurtz and Goldberg, 1971; Sparks and Mays, 1981; Sparks and Hartwich-Young, 1989). When the head is prevented from moving and gaze shifts are accomplished only by saccadic eye movements, the locus of motor activity in the SC is tightly coupled to the vector of the observed saccade. Whether the activity in the SC specifies the amplitude of the observed movement or the amplitude of the desired movement to the initially presented target remains an open question. The nature of the SC motor commands during saccadic adaptation has been addressed by recording motor-related activity during saccadic adaptation (Frens and Van Opstal, 1997; Takeichi et al., 2007). In the Frens and Van Opstal (1997) study, the authors concluded that the locus of SC motor-related activity did not change despite changes in the amplitude of observed saccades over the course of adaptation. In contrast, Takeichi and colleagues (2007) state that their data indicate that saccade amplitude adaptation produces clear changes in discharge at the level of the SC. The latter authors go further and point out that during backward adaptation there was a consistent change in the number of action potentials in the saccade-related burst in the SC. Based on these results, Takeichi et al. (2007) concluded that adaptive plasticity in the saccadic system occurs at the level of the superior colliculus (or that SC activity reflects changes that occur at higher centers).

The experiment described here was designed to determine whether motor activity in the SC systematically changes location as movement amplitudes change during adaptation. Alternatively, the location of activity within the SC motor map could be related to the initial target location and remain unaltered despite significant changes in saccade amplitude. The data presented 
here indicate that there is no change in the locus of SC activity (no remapping of desired saccade vector at the level of the SC) during either backward or forward adaptation.

\section{Materials and Methods}

Preparation of subjects and data acquisition and analysis. Three female rhesus monkeys weighing $4.5-6.0 \mathrm{~kg}$ served as subjects. A scleral coil was implanted for monitoring eye position (Judge et al., 1980) and, during the same aseptic surgery a small head-restraint device was secured to the skull. After full recovery, subjects were trained to make gaze shifts to visual targets. During a second procedure a recording cylinder (David Kopf Instruments) was placed over the SC. All surgical and experimental procedures were approved by the University of Rochester Animal Care and Use Committee, and are in accordance with the National Institutes of Health Guide for the Care and Use of Animals.

During all training and experimental sessions animals were seated with their heads restrained and aligned on the body midline. Subjects sat in the center of $1.2 \mathrm{~m}$ cube that housed 3 pairs of magnetic field coils (CNC Engineering). The four vertical faces of the cube (front-back and leftright) contained two pairs of Helmholtz coils in spatial and phase quadrature (Collewijn, 1977). The current produced in the scleral coil was linearly related to horizontal rotational position of the scleral coil within $\sim 2 \%$. The top and bottom faces of the cube contained a third pair of Helmholtz coils that were used to measure vertical eye position. Visual targets were presented using red $(650 \mathrm{~nm})$ laser diodes attached to 2-axis motorized Fick gimbals (custom built using RGV-100 rotation stages; Newport Corp.) pointed at the inside of a $1.5 \mathrm{~m}$ diameter hemisphere ( 0.5 inch acrylic; Capital Plastics Company Inc.). The center of the hemisphere's vertical face was aligned with the geometric center of the field coil frame. Targets were presented to subject $\mathrm{O}$ using a static array of tri-color LEDs spaced every $2^{\circ}$.

Custom software was used to control behavioral contingencies and acquire eye position data (sampled at $1 \mathrm{kHz}$ and stored for off-line analysis). Standard amplification (Bak Electronics) and filtering techniques were used to isolate and record single neurons from the intermediate and deep layers of the superior colliculus (SC). The SC was identified based on location relative to the stereotactically placed recording chamber, and on the characteristic neural activity observed in the SC. A time-amplitude window (Bak Electronics) was used to isolate action potentials from single neurons. In addition to recording the times at which individual action potentials occurred, the analog spike train was digitized at $25 \mathrm{kHz}$ and used offline to confirm electrical isolation of each neuron.

Behavioral tasks. The two behavioral tasks performed by our subjects (Fig. 1) began identically with the illumination of a central target (T0). Fixation of this target within a $2.5-4^{\circ}$ diameter computer defined window was required for between 350 and $1000 \mathrm{~ms}$ (50 ms increments) in order for the trials to continue. When this interval was completed a second target (T1) was illuminated, but continued fixation of T0 was required for a delay period lasting from 350 to $1000 \mathrm{~ms}$ (50 ms increments). After the delay interval (used to temporally isolate transient visual responses from motor-related SC activity) the T0 target was turned off providing the cue for the subject to direct the line of sight to the T1 target. Saccades toward the T1 target were detected when eye position exceeded the limits of the computer defined window centered on the now invisible T0 target location, and this led to extinction of the T1 target. During "probe" trials (Fig. 1A), saccades continued to completion without further illumination of visual targets and therefore no visual feedback about movement accuracy was available. During "adaptation" trials (Fig. $1 B), 20-40 \mathrm{~ms}$ after saccade detection a third target (T2) was illuminated. During "backward" adaptation, the T2 target was placed on a line between the T0 and T1 targets, and between 40 and $60 \%$ of the distance between them. During "forward" adaptation the T2 target was placed beyond the T1 target such that the T0, T1 and T2 targets fell on the same line. The distance from T0 to T2 was between 1.4 and 1.7 times the distance from T0 to T1. All trials before adaptation began were probe trials. During adaptation, probe trials (both to T1 and to other locations; $\sim 10 \%$ of trials) were randomly interspersed with adaptation trials. When unit isolation was maintained for a sufficient duration a recovery
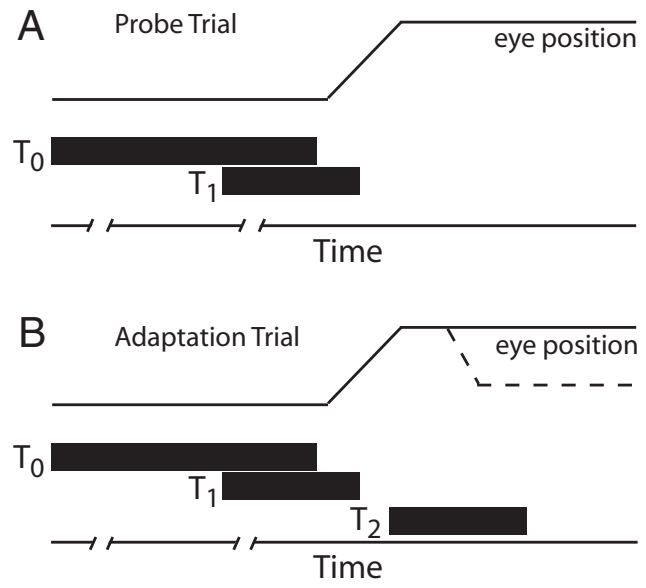

Figure 1. Schematic representation of trial types. The delayed probe trial began with the presentation of the visual target at TO $(\boldsymbol{A})$. After a variable fixation interval (indicated by line breaks along the time axis) a second target was presented. This target could be at the $\mathrm{T} 1$ location or at other locations used to define the movement field of rerecorded cells. Subjects were not permitted to look to the 11 target until target $\mathrm{TO}$ was turned off. Saccade initiation was detected using a position criterion and $\mathrm{T} 1$ was then turned off. The delayed adaptation trials were identical until $20-40 \mathrm{~ms}$ after T1 was turned off. T2 was now illuminated and remained lit until the subject fixated this location for $500 \mathrm{~ms}$. Dotted lines in $\boldsymbol{B}$ indicate the corrective saccade that to the T2 location during backward adaptation.

period followed adaptation. During recovery the T1 target was reilluminated after the primary saccade was made.

Experimental design and rationale. To determine whether there was a shift in location of the active population within the SC during changes in saccade amplitude induced by adaptation, the movement fields of SC neurons were first recorded using the probe saccade task. It is assumed that during control movements when discharge of the recorded neuron is maximal that the cell resides near the center of the active population of motor-related discharge in the SC. A schematic diagram of the SC indicating the location of a hypothetical neuron is shown in Figure $2 \mathrm{~B}$. Also shown here is a representation of the active population of neurons during a $25^{\circ}$ control saccade directed along the horizontal meridian. In $2 \mathrm{~A}$, eye position is plotted as a function of time for two representative saccades. Both are directed along the horizontal meridian. During a $25^{\circ}$ saccade (gray trace in $2 A$ ) the example neuron discharged vigorously (Fig. $2 B$, white circular region at the center of the active population). In contrast, during a $15^{\circ}$ horizontal saccade (black trace in $2 \mathrm{~A}$ ) the discharge of the cell is expected to be much lower. The underlying assumption, schematized in Figure $2 C$, is that the active population of neurons during a $15^{\circ}$ control saccade is centered on a more rostral region of the SC (one that specifies a $15^{\circ}$ movement). As a result the recorded neuron is no longer near the population center but resides at the periphery of active SC neurons.

During saccadic adaptation, the amplitude of the primary saccade to the T1 target changes over time. Two hypotheses about the role of the SC in saccadic adaptation make different predictions about the activity of recorded SC neurons. Hypothesis 1 (Fig. 3A, left) states that the location of the active SC population specifies the amplitude of the observed movement. This hypothesis predicts that, as saccade amplitude declines during backward adaptation, the locus of SC activity will be located in progressively more rostral locations. For example, the SC population will be centered initially at the $25^{\circ}$ locus and then as saccade amplitudes decline activity will be centered at the $18^{\circ}$ site in the SC map (Fig. $3 \mathrm{~A}$, middle). The activity of the recorded neuron (e1) will systematically decline as the active population is centered on more rostral sites. This prediction is illustrated in the right panel of Figure $3 \mathrm{~A}$. In this panel, saccade amplitude is plotted as a function of adaptation trial number, and the shading of the points represents the motor-related discharge of the example cell. As saccade amplitude declines, motor activity declines as well.

The prediction of hypothesis 1 that neural discharge will decline during backward saccadic adaptation is based on placing the $\mathrm{T} 1$ target at a 

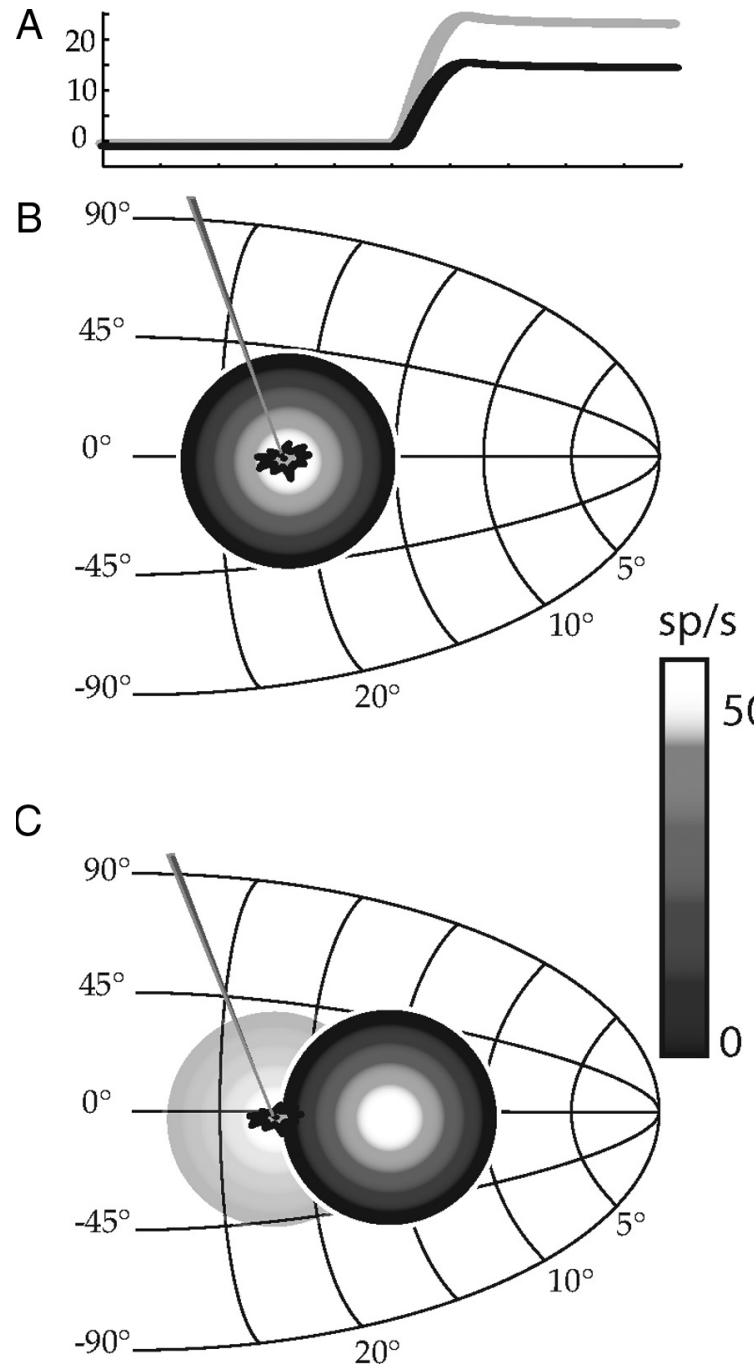

Figure 2. Collicular movement fields. In $\boldsymbol{A}$, horizontal saccade position is plotted as a function of time for two saccades: one to a $25^{\circ}$ target (gray) and the other to a $15^{\circ}$ target (black). In $\boldsymbol{B}$, a schematic diagram of the left SC is illustrated with the hypothetical active population of cells indicated with concentric circles. The location of this population of active cells indicates a motor command specifying a $25^{\circ}$ saccade along the horizontal meridian. Different discharge rates are indicated by different shades (see scale bar). A diagram showing the location of a neuron within this active population is shown. In $\mathbf{C}$, the active population during a $15^{\circ}$ movement is shown (the location of the population during the $25^{\circ}$ saccade is lightly shown for comparison).

location such that the initial (preadaptation) saccade to the target places the recorded cell at the center of the active population. This hypothesis makes the opposite prediction (motor-related activity will systematically increase during adaptation) if the $\mathrm{T} 1$ target is positioned such that the recorded cell is at the rostral edge of the active population at the beginning of backward adaptation (Fig. 3A, electrode e2). Under these conditions as saccade amplitude declines and the active population shifts to more rostral locations the recorded cell is increasingly near the center of the population and its discharge is expected to increase.

The alternative hypothesis (Hypothesis 2; Fig. 3B) states that the locus of motor-related activity in the SC specifies the amplitude and direction of the desired movement to the $\mathrm{T} 1$ target. Changes in saccade amplitude resulting from adaptation arise "downstream" from the SC and so the locus of SC activity remains constant regardless of adaptive changes in saccade amplitude (Fig. 3B, middle). If this were the case, discharge of SC neurons will remain unaltered despite changes in saccade amplitude due to adaptation. As shown on the right of Figure $3 B$, discharge of this example cell remains high because the cell remains at the center of the active population throughout adaptation. If the T1 target is located such that the cell is initially at the rostral edge of the active population (Fig. $3 B-\mathrm{e} 2$ ), this hypothesis predicts that neural activity will remain low and unaltered despite declining saccade amplitudes.

The predictions of these two alternative hypotheses during forward adaptation are presented in Figure 3, $C$ and $D$. Using the same layout, if activity in the SC changes systematically as saccade amplitudes increase, neural activity of a cell at position el will systematically increase during adaptation (Fig. 3C). In contrast the alternative predicts that during forward adaptation, no change in SC activity will occur since the SC specifies a desired movement to the $\mathrm{T} 1$ location throughout adaptation (Fig. 3D). Note that it is possible that forward and backward adaptation are accomplished using different mechanisms (Ethier et al., 2008).

Data analysis. All off-line analyses were accomplished using Matlab (MathWorks). Saccade beginning and end were automatically identified when movement velocity exceeded and fell below $20 \%$ s. Movement amplitude was defined as the difference in horizontal and vertical position from beginning to end of the identified movement. Vectorial amplitude was calculated as the square root of the sum of the squared horizontal and vertical amplitudes. Motor related activity was defined as the action potentials that occurred between $60 \mathrm{~ms}$ before saccade onset until the end of the saccade; the number of action potentials was divided by the duration of this epoch to calculate discharge rates. The mean discharge of SC neurons observed during adapted movements to the T1 target and during amplitude- and direction-matched control movements was compared using a Student's $t$ test $(p<0.05)$.

\section{Results}

Activity from 50 neurons was electrically isolated in the deeper SC layers in three rhesus monkeys (subjects $\mathrm{U}, \mathrm{Q}$, and $\mathrm{O}$ ) and was recorded for a sufficient period to assess neural activity before, during and after saccadic adaptation. Thirty-seven of these 50 neurons were recorded during backward adaptation (Table 1). First, the movement field of each cell was characterized. Placement of the T1 and T2 targets followed. For 21 neurons recorded during backward adaptation ( 11 from subject Q, 10 from subject $\mathrm{U}$; standard typeface in Table 1), T1 target locations were selected such that the amplitude and direction of control saccades to this location placed the recorded neuron near the rostral edge of the active population of SC motor related activity (see Fig. $3 A, B$, electrode position e2). In the remaining 16 backward adaptation sessions ( 6 from $U$ and 10 from Q; italics in Table 1) the T1 target was located such that the recorded cell was near the center of the active population during control saccades to the T1 location (for example, see Fig. $3 A, B$, electrode position e1). Thirteen neurons were recorded during forward adaptation (Table 2; 8 from O, 5 from U). In 12 of 13 cases, the location of T1 was selected such that the amplitude and direction of control saccades placed the recorded neuron at the caudal edge of the active population (Fig. $3 C, D$, electrode location e1). In one example (U21 in Table 2: italics), T1 was placed such that the recorded neuron was at the center of the active population during control saccades (Fig. $3 C, D$, electrode location e2).

\section{Backward adaptation}

Figure $4 \mathrm{~A}$ illustrates the target locations (light gray circles) used during recording the preadaptation movement field of a neuron (U2) from the right SC. The location of the fixation target (T0) and the initial target used during adaptation trials (T1) are also shown in $4 \mathrm{~A}$. The movement field data collected using probe trials to the targets indicated are superimposed on the same panel. As shown there was strong motor related activity (discharge rates $>400 \mathrm{sp} / \mathrm{s}$ ) for leftward saccades $\sim 12^{\circ}$ in amplitude directed along the horizontal meridian. As larger amplitude movements were made, motor related activity was less vigorous 
and discharge rates declined to $<150 \mathrm{sp} / \mathrm{s}$ for leftward movements of $\sim 20^{\circ}$. Movements made during preadaptation probe trials to the T1 location (points encircled with black) evoked little motor related activity; the cell was presumably on the outskirts of the active population during movements of this amplitude and direction. Examples of individual trials for 3 sets of movements directed along the horizontal meridian are presented in Figure $4 B$. For each set of 5 trials, horizontal eye position is plotted as a function of time in the top. Mean amplitudes for these example trials were $11.7^{\circ}$ (red), $15.8^{\circ}$ (green) and $21.3^{\circ}$ (blue). Using the corresponding colors, the rasters and histograms $(5 \mathrm{~ms}$ bins), aligned on the start of the primary saccade, are also shown (4C). As anticipated based on the movement field, this cell discharged a vigorous motor burst during movements $\sim 12^{\circ}$ (red) and a much reduced motor-related burst during movements of $\sim 21^{\circ}$ (blue).

The preadaptation trials that form the movement field shown in Figure 4 are also plotted in Figure $5 A$ (circles). In $5 A$, however, the vectorial amplitude of each saccade is plotted as a function of the order in which the trial occurred (color bar is given in 5C). The first 175 trials were preadaptation trials to a variety of targets. Two subsets of preadaptation trials are highlighted. The first set having amplitudes between 20 and $22^{\circ}$ are movements made during probe trials to $\mathrm{T} 1$ (data points encircled with gray). The second set of highlighted trials had amplitudes between 12 and $15^{\circ}$ (encircled with black). This second set of trials was selected to compare cell discharge before and after adaptation. In Figure $5 A$, motor related discharge of this cell is illustrated during adaptation trials (star symbols) and during probe trials to T1 that were randomly interleaved during adaptation (asterisk symbols). As shown in Figure $5 A$, the discharge rate of motor related activity did not change over the course of $\sim 500$ adaptation trials despite the large change in saccade amplitude. Single trial examples of movements that occurred at the start (red), in the middle (green) and near the end (blue) of the adaptation process are shown in 5B. Horizontal eye position is plotted as a function of time for each set of 5 trials and below are the rasters for individual trials and averaged histograms (5 ms bins) aligned on the beginning of each saccade.

Comparing movements made before and after adaptation, and as shown in $5 \mathrm{~A}$ and $\mathrm{B}$, the $\sim 30 \%$ reduction in saccade amplitude during movements to $\mathrm{T} 1$ produced little or no change in the neural discharge of the recorded cell. As a result movements

\section{Backward Adaptation}

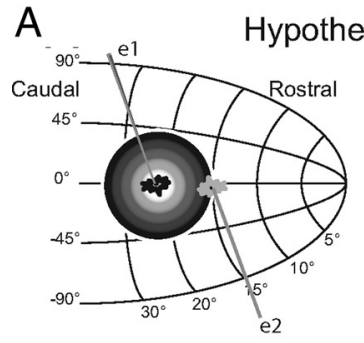

B
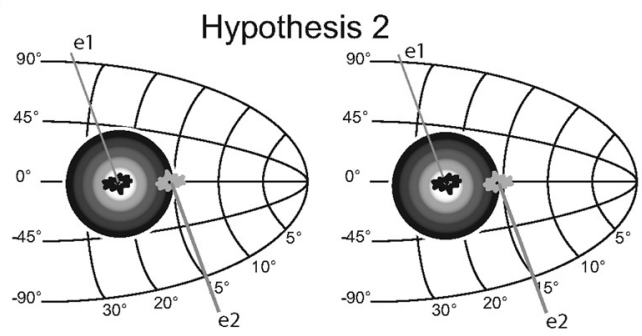

Forward Adaptation
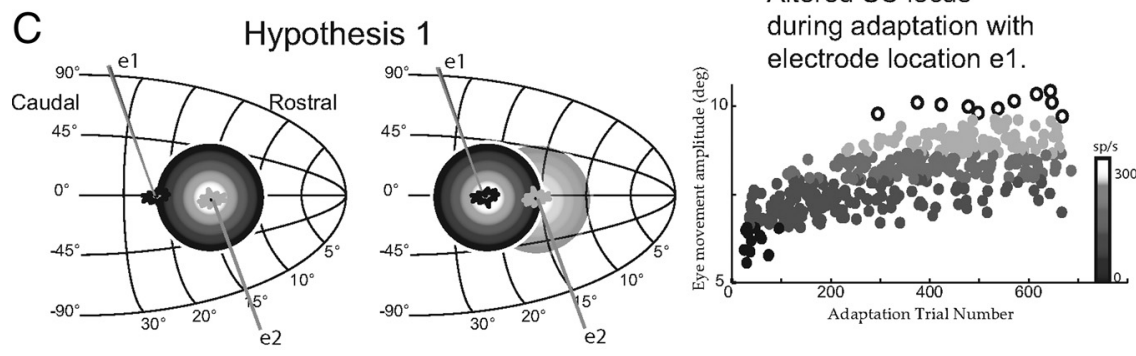

Altered SC locus during adaptation with electrode location $\mathrm{e} 1$.
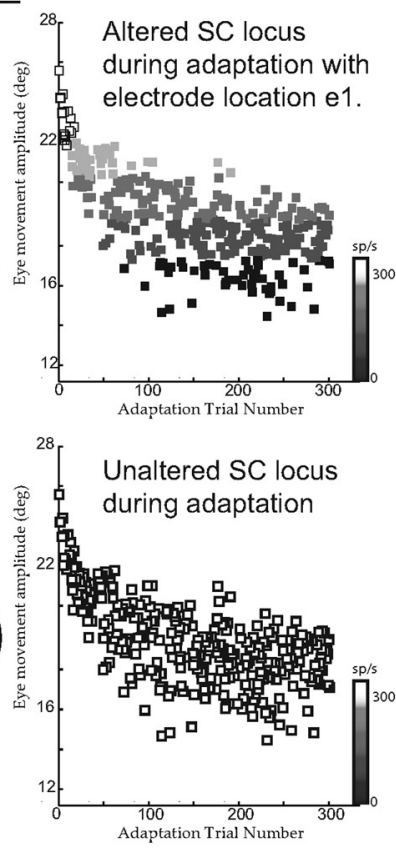

D

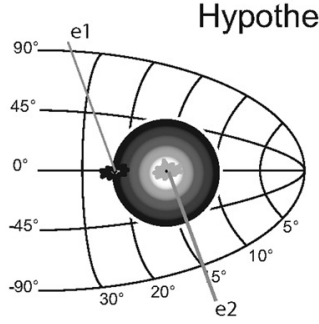

Figure 3. Predictions of alternative hypotheses. As in Figure 2, motor activity on the SC map is presented as concentric circles (white is high activity; black low activity). Recorded neurons are indicated with drawn electrodes and cell bodies. In A, the active population during a $25^{\circ}$ horizontal control movement, and a cell located at the population center (electrode position e1) and a second located at the rostral edge of the population (position e2). Hypothesis 1 posits a change in the locus of SC activity during adaptation. This is indicated in the middle. If activity were recorded from a cell at electrode position e1, at the start of adaptation discharge rates would be high. This is shown in the right-most panel that plots saccade amplitude as a function of the number of adaptation trails and the shading of points indicates the discharge rate of a hypothetical cell. Early in adaptation the cell is at the center of the active population and discharges vigorously. As movement amplitudes decline the active population is presumed to move to more rostral sites and activity is predicted to decline. $\boldsymbol{B}$ presents the predictions of the alternative hypothesis (hypothesis 2) using the same layout. This hypothesis predicts no change in location of the active population in the SC. The recorded neuron (position e1) remains at the center of the active population throughout the adaptation process. Thus at the end of adaptation, movements that are much smaller than control movements to T1 are associated with high discharge rates. In $\boldsymbol{C}$ and $\boldsymbol{D}$, the predictions of these two alternative hypotheses are presented during forward adaptation. The layout is the same as in $\boldsymbol{A}$ and $\boldsymbol{B}$. that had been associated with very vigorous discharge before adaptation were associated with low firing rates during matched movements that occurred after adaptation. When movement field data from the end of the adaptation process are superim- 
Table 1. Comparing discharge of SC neurons before and after backward adaptation

\begin{tabular}{|c|c|c|c|c|c|c|c|c|c|c|c|c|c|c|c|c|c|c|c|c|c|c|}
\hline \multirow[b]{3}{*}{ Cell ID } & \multirow{3}{*}{$\begin{array}{l}\text { Adaptation } \\
\text { gain }\end{array}$} & \multicolumn{7}{|c|}{$\begin{array}{l}\text { Preadaptation } \\
\text { movements to } \mathrm{T} 1\end{array}$} & \multicolumn{7}{|c|}{$\begin{array}{l}\text { Postadaptation } \\
\text { movements to T1 }\end{array}$} & \multicolumn{7}{|c|}{$\begin{array}{l}\text { Preadaptation movements matched to } \\
\text { postadaptation amplitude and direction }\end{array}$} \\
\hline & & \multicolumn{2}{|c|}{ Spikes/s } & \multicolumn{2}{|c|}{ No. of spikes } & \multicolumn{2}{|c|}{ Duration } & \multirow[b]{2}{*}{$n$} & \multicolumn{2}{|c|}{ Spikes/s } & \multicolumn{2}{|c|}{ No. of spikes } & \multicolumn{2}{|c|}{ Duration } & \multirow[b]{2}{*}{$n$} & \multicolumn{2}{|c|}{ Spikes/s } & \multicolumn{2}{|c|}{ No. of spikes } & \multicolumn{2}{|c|}{ Duration } & \\
\hline & & Mean & (SD) & Mean & (SD) & Mean & (SD) & & Mean & (SD) & Mean & (SD) & Mean & (SD) & & Mean & (SD) & Mean & (SD) & Mean & (SD) & $n$ \\
\hline Q1 & 0.66 & 86.0 & (19.6) & 8.8 & (2) & 42.4 & (2.3) & 15 & $85.7^{*}$ & (22.2) & $8.6^{*}$ & (2.2) & 40.9 & (4.5) & 80 & 260.6 & (38.2) & 24.3 & (3.4) & 33.2 & (1.7) & \\
\hline Q2 & 0.68 & 53.2 & (37.4) & 6.0 & $(4.2)$ & 52.5 & $(0.7)$ & 2 & $69.8^{*}$ & (28.6) & $7.6^{*}$ & (3.2) & 49.1 & (4.1) & 25 & 142.1 & (13.3) & 14.0 & (1.4) & 38.5 & $(0.7)$ & \\
\hline Q3 & 0.77 & 130.3 & (20.4) & 16.3 & (2.4) & 65.4 & $(4.6)$ & 39 & $118.7^{*}$ & (18.7) & $14.6^{*}$ & (2.3) & & (3.6) & 40 & 172.9 & (18.9) & & & 55.8 & (3.9) & \\
\hline Q4 & 0.72 & 115.3 & $(50.9)$ & 13.3 & (5.9) & 55.2 & (2.2) & 9 & $104.2^{*}$ & (59.1) & $11.3^{*}$ & (6.3) & & (4) & 72 & 225.2 & $(40.1)$ & & (6.7) & 42.0 & (2.4) & \\
\hline Q5 & 0.78 & 154.1 & (23.1) & 17.0 & (2.4) & 50.4 & (1.4) & 7 & $137.6^{*}$ & (22) & $15.1^{*}$ & (2.3) & 49.5 & (3.3) & 37 & 192.7 & 8.9) & & (3) & 42.1 & (2.3) & \\
\hline Q6 & 0.69 & 42.4 & $(14.5)$ & 4.6 & (1.6) & 48.1 & (4.1) & 10 & $57.9^{*}$ & (27.4) & $6.1^{*}$ & (2.8) & 45.2 & (4.3) & 51 & 243.6 & (35.8) & 23.5 & (3) & 36.8 & (3.6) & 16 \\
\hline Q7 & 0.82 & 201.5 & (31.3) & 20.7 & (3.1) & 42.7 & (2) & 24 & $183.7^{*}$ & (25.4) & $18.9^{*}$ & (2.5) & 42.8 & (2.9) & 30 & 294.4 & (32.6) & 28 & (2.6) & 37.0 & (2.8) & \\
\hline Q8 & 0.70 & 133.5 & (27.6) & 13.1 & (2.6) & 38.4 & (2.6) & 27 & $90^{*}$ & $(25.6)$ & $9.1^{*}$ & (2.5) & 40.8 & (4.1) & 74 & 222.3 & (29.8) & & & 33.7 & (2.4) & 2 \\
\hline Q9 & 0.73 & 165.2 & (49.5) & 16.4 & (4.7) & 39.8 & (2.2) & 24 & $133.2^{*}$ & (44.6) & $13.5^{*}$ & (4.3) & 42.2 & (4.6) & 36 & 320.5 & $(60.2)$ & 29.4 & (5.3) & 32.0 & (1.7) & \\
\hline Q10 & 0.63 & 95.3 & (21.1) & 9.9 & (2) & 43.8 & (2.8) & 20 & $67.8^{*}$ & $(20.3)$ & $7^{*}$ & (2.1) & 43.6 & (5.3) & 79 & 219.1 & (30.9) & 20 & (2. & 32.6 & (1.1) & \\
\hline Q11 & 0.76 & 74.8 & (39.8) & 8.5 & $(4.5)$ & 53.4 & (2.7) & 32 & $50.7^{*}$ & $(35.9)$ & $5.6^{*}$ & & & (3.4) & 50 & 53.8 & & & & 41.2 & (2.9) & \\
\hline Q12 & 0.70 & 247.4 & (31) & 24.5 & (8.6) & 48.9 & (4.6) & 10 & $229.1^{*}$ & (29.7) & $25^{*}$ & (3.5) & 49.2 & (4.9) & 30 & 133.7 & 1.8) & 13 & & 38.2 & (2) & \\
\hline Q13 & 0.63 & 249.6 & (20.1) & 29.4 & (2.2) & 57.7 & (3.4) & 11 & $265.6^{*}$ & (21.1) & $30^{*}$ & (2.3) & 53.4 & (7.8) & 45 & 157.6 & (45.4) & 15.4 & (4.5) & 37.6 & (0.9) & \\
\hline Q14 & 0.72 & 309.7 & (41.6) & 33.9 & (4.3) & 49.6 & (2.3) & 19 & $277.9^{*}$ & (38.6) & $30.5^{*}$ & (4.1) & 49.8 & (4.5) & 58 & 192.8 & (18) & 19 & (2) & 39.8 & (1.7) & \\
\hline Q15 & 0.70 & 225.1 & (12.4) & 27.7 & (2.1) & 62.9 & (4.8) & 16 & $272.6^{*}$ & (21.2) & $31.5^{*}$ & (2.6) & 55.5 & (6) & 39 & 185.0 & (20.9) & & (2.5) & 46.9 & (3.8) & \\
\hline Q16 & 0.75 & 142.2 & (20.1) & 17.3 & (2.7) & 61.9 & (5.3) & 32 & $141.2^{*}$ & (25) & 16. & (3) & 56.2 & (4.1) & 36 & 97.4 & (15 & & (1.9) & 47.3 & (4.2) & \\
\hline Q17 & 0.71 & 137.9 & (28.1) & 16.4 & (3.3) & 59.3 & (3.3) & 19 & $149.9^{*}$ & (21.7) & $16.8^{*}$ & (2.4) & 52. & (4.6) & 76 & 42.7 & & & (1. & 41.6 & (4) & \\
\hline Q18 & 0.76 & 128.5 & (20.4) & 14.7 & (2.6) & 53.9 & (4) & 34 & $150.4^{*}$ & (25.6) & $16.5^{*}$ & (2.8) & 49.8 & (4.1) & 152 & 27.1 & (17.3) & 2.7 & (1.8) & 39.9 & (2.5) & 49 \\
\hline Q19 & 0.69 & 256.6 & (29.3) & 25.5 & (2.9) & 39.4 & (2.6) & 16 & $270.5^{*}$ & (30.7) & $27.5^{*}$ & (3.2) & 41.8 & (4.7) & 25 & 141.9 & (29.1) & 13.4 & (2.8) & 34.1 & (2.5) & 22 \\
\hline Q20 & 0.64 & 175.7 & (14.3) & 21.0 & (1.8) & 59.5 & (1.8) & 8 & $160.6^{*}$ & (20.1) & $18^{*}$ & (2.2) & 52.2 & (3.2) & 36 & 48.6 & (8.4) & 5.0 & $(0.8)$ & 43.0 & (4.8) & \\
\hline Q21 & 0.81 & 225.3 & (26.6) & 25.4 & (3) & 52.7 & (2.7) & 36 & $222.3^{*}$ & (23.2) & $24.7^{*}$ & (2.3) & & (4.5) & 18 & 94.8 & (37.7) & 9.6 & (4) & 40.2 & (2.9) & \\
\hline U1 & 0.63 & 56.1 & $(44.2)$ & 6.0 & (4.7) & 47.5 & (1.7) & 4 & & (32.3) & & & & (3.4) & 107 & 216.4 & 8.2) & 21. & (3.6) & 8.3 & (2.4) & \\
\hline J2 & 0.68 & 123.9 & (11.2) & 14.7 & (1.4) & 58.3 & (1.6) & 6 & $126.6^{*}$ & (26.9) & $14.2^{*}$ & (2.9) & 52.3 & (5.4) & 70 & 351.6 & (29.5) & 37.8 & (3.1) & 47.7 & (1.5) & \\
\hline U3 & 0.53 & 130.2 & $(64.5)$ & 13.7 & (6.3) & 46.6 & $(4.3)$ & 16 & $159.1^{*}$ & (34.2) & $16^{*}$ & (3.4) & 41.3 & (8.7) & 23 & 296.7 & (50.7) & 27.4 & (5) & 32.2 & (2.9) & \\
\hline U4 & 0.72 & 115.4 & (48.6) & 13.3 & (5.3) & 56.2 & (5.5) & 20 & $124.1^{*}$ & (22.1) & $14.2^{*}$ & (2.4) & 54.4 & (4.6) & 29 & 298.7 & (23.7) & 29.0 & $(2.2)$ & 37.2 & (5.6) & \\
\hline U5 & 0.57 & 5.1 & $(7.2)$ & 0.5 & $(0.7)$ & 39.5 & (2.1) & 2 & $7.9^{*}$ & (15.1) & $0.7^{*}$ & (1.4) & & (3.1) & 46 & 308.8 & (41.1) & 27.8 & (3.3) & 30.0 & (1.4) & \\
\hline U6 & 0.63 & 177.7 & (54.4) & 19.5 & (6) & 49.8 & $(0.5)$ & 4 & $126.3^{*}$ & (24.3) & $13.4^{*}$ & (2.6) & 46.2 & (2.6) & 69 & 294.6 & (23.1) & 29.1 & (2.5) & 38.9 & (3.4) & \\
\hline U7 & 0.64 & 108.9 & (28.8 & 11.8 & (2.8) & 48.5 & (3.7) & 4 & & (29. & 14.7 & & & (2.7) & 77 & 280.2 & (32.2) & 28 & (2. & 40.0 & (1.4) & \\
\hline U8 & 0.82 & 197.1 & (17.4) & 24.9 & (2.1) & 66.2 & $(2.2)$ & 21 & $151.2^{*}$ & (39.7) & $18.7^{*}$ & (4.9) & 63.7 & (2) & 29 & 210.3 & (27.3) & 25.2 & (3.3) & 59.8 & (2.2) & \\
\hline U9 & 0.70 & 22.6 & (8.1) & 2.6 & $(0.9)$ & 57.2 & (1.7) & 11 & $47.7^{*}$ & (21.9) & $5.3^{*}$ & (2.4) & 50.6 & (1.9) & 98 & 125.7 & (49) & 13.1 & $(4.8)$ & 45.1 & (6.1) & 13 \\
\hline U10 & 0.75 & 15.2 & (15.4) & 1.8 & (1.8) & 59.2 & (2.1) & 10 & $58.4^{*}$ & (28.6) & $6.7^{*}$ & (3.3) & 55.0 & (3.4) & 52 & 193.2 & (45.7) & 21.8 & (4.1) & 53.8 & (8.3) & 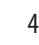 \\
\hline U11 & 0.74 & 344.3 & $(47.5)$ & 39.1 & (5.8) & 53.8 & (8.3) & 17 & $372.9^{*}$ & $(37.9)$ & $42.1^{*}$ & (4.4) & 53.0 & (5.2) & 64 & 261.8 & $(60.9)$ & 25.5 & (6.1) & 37.1 & (2.1) & 1. \\
\hline U12 & 0.53 & 503.1 & (32.2) & 60.3 & (4.3) & 59.9 & (3.4) & 12 & $459.6^{*}$ & (56.3) & $51.8^{*}$ & (6.7) & 52.6 & (6.9) & 71 & 216.9 & (96.6) & 22.5 & (10.7) & 42.4 & (5.6) & 27 \\
\hline U13 & 0.54 & 405.3 & $(44.3)$ & 47.5 & (5.1) & & (2.8) & 15 & & (84.7) & & & & (5.9) & 47 & 180.2 & (73.6) & & (7.9) & 41.8 & (3.1) & 37 \\
\hline U14 & 0.63 & 280.9 & $(34.2)$ & 38.1 & $(4.9)$ & 75.5 & (2.2) & 12 & $275.1^{*}$ & (29.4) & $36.5^{*}$ & (3.6) & 73.0 & (7.3) & 65 & 122.8 & (51.3) & 15.1 & (6.6) & 62.4 & (2.4) & \\
\hline U15 & 0.82 & 343.3 & $(29.5)$ & 41.6 & $(4.2)$ & 61.1 & (2) & 8 & $377.4^{*}$ & $(37.6)$ & $44.6^{*}$ & $(4.5)$ & 58.2 & (1.9) & 31 & 297.3 & $(48.5)$ & 33.6 & (4.6) & 53.6 & (5.9) & \\
\hline U16 & 0.72 & 163.2 & (25.6) & 21.5 & (3.3) & 71.8 & (1.3) & 4 & $172.2^{*}$ & (28.5) & $21.3^{*}$ & (3.4) & 64.0 & (2.1) & 77 & 88.6 & (26.6) & 9.2 & (2.6) & 44.5 & (9.7) & 11 \\
\hline
\end{tabular}

Beginning from the left, the table presents the cell identification number, and the gain of the backward adaptation (postadaptation amplitude/preadaptation amplitude). For preadaptation movements to the T1 location, postadaptation movements when T1 was illuminated, and preadaptation movements that were matched for amplitude and direction with the postadaptation movement vectors, mean discharge rates (and standard deviation), mean number of spikes (SD) in measurement window ( $60 \mathrm{~ms}$ before saccade onset to saccade end), mean duration of saccades (SD), and number of trials contributing to the means are shown. Discharge rates during postadaptation were compared to matched vector preadaptation movements (Student's t test). Number of spikes in the burst during postadaptation saccades was compared with matched vector preadaptation movements (Student's $t$ test). Asterisks indicate that in every case discharge rates and number of spikes were statistically significantly different $(p<0.05)$. Italicized entries indicate electrode position e1 from Fig. 3; standard typeface indicates electrode position e2.

posed on the preadaptation movement field (Fig. 5C) the striking differences in discharge for movements of the same amplitude and direction are clear. The inset in $5 C$ expands the region of the movement field that includes the postadaptation movements to the T1 target location. Note that some trials are adaptation trials (stars) whereas others are probe trials (asterisks). The black rectangle contains all the trials used for subsequent preadaptation and postadaptation comparison (see below). Six preadaptation and six postadaptation trials were randomly selected from this group for display in Figure 5D. In the upper section horizontal eye position is plotted as a function of time for each of these 12 trials. Trials shown in blue were postadaptation trials made in response to presentation of the T1 target whereas those shown in red are preadaptation trials matched for amplitude and direction. The rasters and histograms shown below emphasize the large difference in discharge rates before (red) and after (blue) adaptation.

The alternative hypotheses make predictions about the discharge of SC neurons based on the amplitudes of observed sac- cades. These differ most when comparing postadaptation saccades having amplitudes that differed most from preadaptation control trials to T1. For statistical comparison trials having the largest adaptive change in amplitude ( $\geq 90 \%$ of the maximal change observed) were selected and their amplitudes and directions matched to trials collected before adaptation. In addition, preadaptation trials to the T1 target were compared with this same group of postadaptation movements. For this example neuron (U2 in Table 1), there were 6 preadaptation probe trials to T1 and these were associated with mean (SD) discharge rates of 123.9 (11.2) sp/s. Seventy postadaptation trials were selected for comparison and these were associated with a mean discharge rate of 126.6 (26.9) sp/s. Thirteen preadaptation trials with matched amplitudes and directions were compared with the postadaptation trials. For these sets of movements having nearly identical metrics, preadaptation trials had a mean discharge rate of 351.6 (29.5) sp/s.

Another example (U12) of SC activity during backward adaptation is provided in Figure 6. In this example, the T1 target was 


\begin{tabular}{|c|c|c|c|c|c|c|c|c|c|c|}
\hline \multirow[b]{3}{*}{ Cell ID } & \multirow[b]{3}{*}{ Adaptation gain } & \multicolumn{3}{|c|}{ Preadaptation movements to T1 } & \multicolumn{3}{|c|}{ Postadaptation movements to T1 } & \multicolumn{3}{|c|}{$\begin{array}{l}\text { Preadaptation movements matched to } \\
\text { postadaptation amplitude and direction }\end{array}$} \\
\hline & & \multicolumn{3}{|c|}{ Spikes/s } & \multicolumn{3}{|l|}{ Spikes/s } & \multicolumn{3}{|c|}{ Spikes/s } \\
\hline & & Mean & (SD) & $\mathrm{n}$ & Mean & (SD) & $n$ & Mean & (SD) & $n$ \\
\hline 01 & 1.32 & 76.2 & $(23.4)$ & 12 & $68.1^{*}$ & (29.9) & 33 & 121.6 & (15) & 7 \\
\hline $\mathrm{O}_{2}$ & 1.46 & 63.5 & $(12.8)$ & 6 & $74.9^{*}$ & $(17)$ & 28 & 121.1 & $(9.6)$ & 4 \\
\hline 03 & 1.27 & 84.7 & (14.1) & 9 & $85.7^{*}$ & (16.6) & 47 & 170.5 & $(25.3)$ & 15 \\
\hline 04 & 1.58 & 74.8 & $(19.3)$ & 12 & $103^{*}$ & $(16.7)$ & 59 & 177.8 & $(0.9)$ & 4 \\
\hline 05 & 1.44 & 148.7 & $(39.4)$ & 17 & $132.1^{*}$ & $(26.3)$ & 31 & 255.6 & $(35.6)$ & 5 \\
\hline 06 & 1.41 & 142.6 & (73.3) & 22 & $120.6^{*}$ & (20.8) & 31 & 221.8 & $(25.2)$ & 9 \\
\hline 07 & 1.27 & 140.7 & $(19.2)$ & 4 & $147.8^{*}$ & $(23)$ & 12 & 178.5 & $(9.7)$ & 4 \\
\hline 08 & 1.29 & 67.7 & $(23.2)$ & 14 & $95.6^{*}$ & (33.8) & 7 & 236.5 & $(20.7)$ & 3 \\
\hline U17 & 1.44 & 79.0 & $(35.2)$ & 5 & $71.1^{*}$ & $(42.8)$ & 67 & 287.6 & (106.1) & 3 \\
\hline U18 & 1.24 & 33.7 & (13) & 18 & $25.6^{*}$ & (13) & 16 & 94.0 & (19.4) & 8 \\
\hline U19 & 1.14 & 11.1 & (11.4) & 12 & $12.7^{*}$ & $(12.7)$ & 24 & 85.3 & (36.6) & 20 \\
\hline U20 & 1.23 & 29.7 & $(22.2)$ & 24 & $27.3^{*}$ & (11.3) & 29 & 75.3 & (39.5) & 10 \\
\hline U21 & 1.37 & 131.8 & (57.4) & 12 & $141.8^{*}$ & (33.2) & 22 & 29.8 & (43.7) & 4 \\
\hline
\end{tabular}

Table layout is the same as described in Table 1. Discharge rates and number of spikes during postadaptation were compared with matched vector preadaptation movements. Asterisks indicate that in every case discharge rates and number of spikes were statistically significantly different $(p<0.05)$. Italicized entries indicate electrode position e1 from Fig. 3; standard typeface indicates electrode position e2.

placed near the center of the cell's movement field (Fig. 3, electrode position e1). In $6 \mathrm{~A}$ the vectorial amplitude of saccades is plotted as a function of trial number for 1000 trials. As in Figure 5, the leftmost 175 trials shown in Figure 6 were probe trials to a variety of targets presented before adaptation was started. During movements to $\mathrm{T} 1$ and nearby targets, the cell discharged at a high rate ( $>400$ spikes/s; color bar in $6 B$ ). Once adaptation trials began, saccade amplitude declined from $\sim 22^{\circ}$ to $\sim 11^{\circ}$. Even though saccade amplitudes were reduced by nearly $50 \%$, motorrelated discharge remained virtually unchanged. As a result, when the postadaptation movement field data are superimposed on the preadaptation data (Fig. 6B) there is a clear difference between the high discharge rates after adaptation and the much lower rates seen before adaptation during movements having similar amplitudes and directions (see inset in 6B). After 750 trials, recording of motor-related discharge continued during recovery. The symbols in Figure $6 \mathrm{~A}$ during recovery outlined in black are probe trials to $\mathrm{T} 1$ during which the $\mathrm{T} 1$ target was reilluminated 100-200 ms after the end of the primary saccade. During recovery, saccades to the $\mathrm{T} 1$ target increased in amplitude over time. Neural discharge continued to be unaltered during recovery compared with preadaptation and postadaptation movements to T1. Detailed comparisons of these and the other 35 sites are given in Table 1.

Table 1 presents data from the 37 neurons recorded during backward adaptation. The left-most column provides the subject identifier and cell number; standard typeface indicates data collected when electrodes were placed in position e 2 in Figure 3 (i.e., initially the recorded cell is at the rostral edge of the active population of SC neurons). Table entries that are italicized indicate data collected using electrode position el (the recorded cell was at the center of the active population at the beginning of adaptation). The second column provides the adaptation gain (postadaptation amplitude/preadaptation amplitude). Mean discharge rates (SEM and number of trials included in the analysis) of the recorded cell are shown during preadaptation movements to the $\mathrm{T} 1$ target, postadaptation movements to T1, and preadaptation movements matched to the postadaptation amplitude and direction. Comparing postadaptation trials with amplitude-matched preadaptation movements (right-most two columns) discharge rates for all examples in our sample were significantly different $(p<0.05)$. Despite having nearly identical amplitudes and di- rections, movements before and after adaptation were associated with markedly different discharge. There were no counter examples observed in our 37 neurons from two monkeys. In every case, data were inconsistent with remapping of the collicular locus of activity as amplitudes changed during saccadic adaptation.

\section{Forward adaptation}

For technical reasons, evaluating the locus of SC activity is more difficult during forward compared with backward adaptation. First, the change in saccade amplitude during forward adaptation is generally smaller than comparable backward adaptation (for review, see Hopp and Fuchs, 2004). Second, the rate of forward adaptation is slower requiring longer maintenance of electrically isolated SC neurons. And third, corrective saccades during forward adaptation are in the same direction as the primary saccade. It is important to assess only activity related to the primary movement. We recorded from 13 SC neurons during forward adaptation and determined whether there was evidence of remapping of the saccadic command (Hypothesis 1; Fig. 3C) or whether the locus of SC activity remained unchanged during forward adaptation (Hypothesis 2; Fig. 3D). Figure 7 provides an example of a neuron $(\mathrm{O} 3)$ recorded during forward adaptation. The layout of the figure is the same as that in Figures 5 and 6. A plots the vectorial amplitude of the primary saccade as a function of the trials presented. Before adaptation the neuron discharged vigorously for saccades $\sim 17^{\circ}$, and during smaller amplitude movements discharged much less vigorously. Saccade amplitude increased from $\sim 12^{\circ}$ to $\sim 16^{\circ}$ over the course of $>600$ adaptation trials. Average discharge rate of the recorded neuron did not change. In $B$, the preadaptation movement field is shown with the locations of T0, T1 and T2 illustrated as well. The postadaptation trials are superimposed on the preadaptation movement field within the gray box and expanded for easier viewing in the inset. The discharge rate after adaptation is clearly lower than it was during the preadaptation movements having the same amplitudes and directions. As illustrated previously during backward adaptation, during forward adaptation the locus of SC motorrelated activity appears to be unaltered despite observed changes in saccade amplitude. These data are inconsistent with the hypothesis that saccade vectors are "remapped" at the level of the SC. Similar results were observed for all recorded neurons in the sample. 

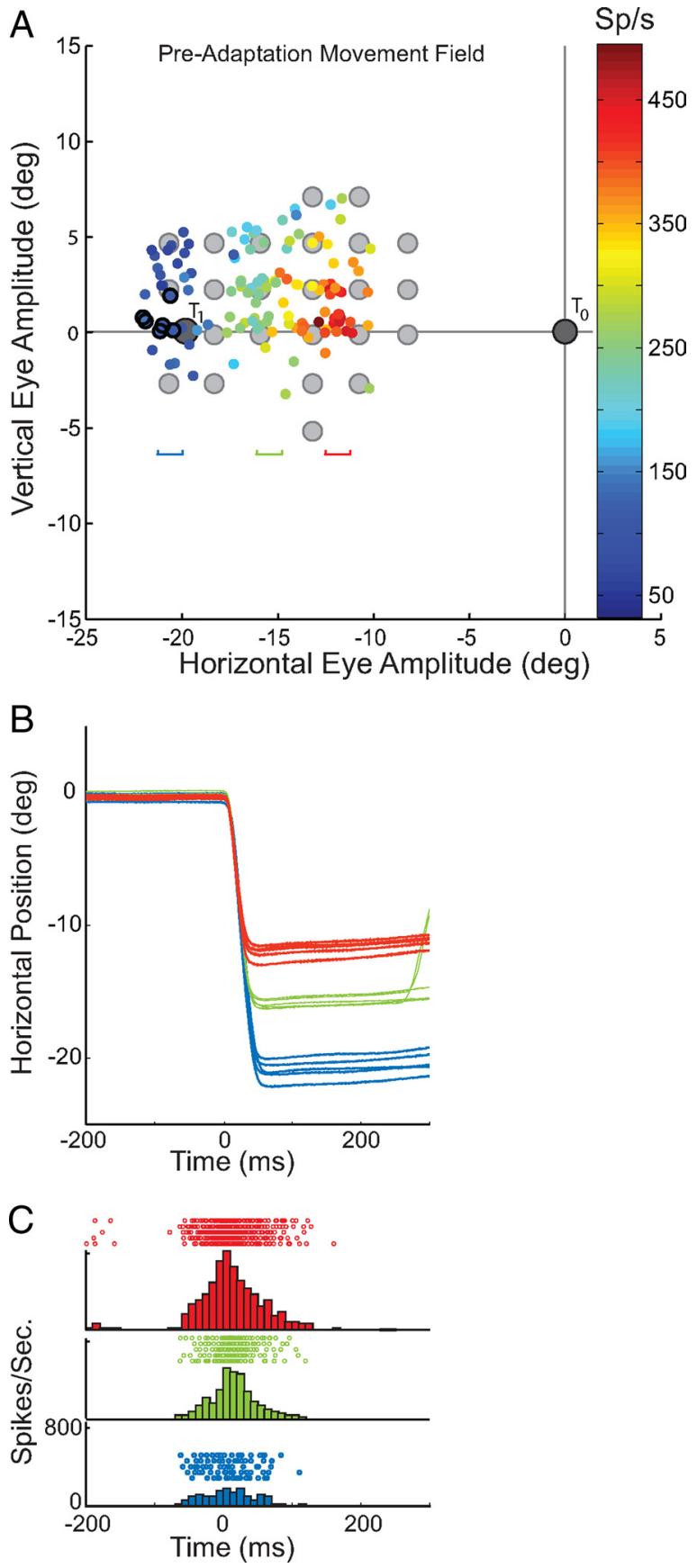

Figure 4. Preadaptation movement field. $\boldsymbol{A}$ plots the preadaptation movement field for neuron U2. Gray filled symbols indicate target locations including the T1 location. Data points encircled in black are preadaptation trials to the T1 location. Three groups $(n=6)$ of movements having different amplitudes are selected for display and horizontal position is plotted as a function of time in $\boldsymbol{B}$. Color codes indicate the region of the movement field from which these are extracted. In $\boldsymbol{C}$, rasters for each trial, and histograms for each group aligned on movement beginning are illustrated for comparison.

Table 2 summarizes the results during forward adaptation. Mean $( \pm \mathrm{SD})$ discharge rates during preadaptation movements to the $\mathrm{T} 1$ location (third column from the left), mean discharge during postadaptation movements to $\mathrm{T} 1$, and discharge during control movements matched for amplitude and direction to the postadaptation saccade vectors are shown. In each case, mean discharge rates during postadaptation movements to T1 were statistically different from discharge rates during amplitude and direction matched preadaptation saccades $(p<0.05)$. These data are inconsistent with the hypothesis that there is a remapping of the saccade vector command at the level of the SC during forward adaptation. The locus of motor activity in the SC appears to encode the desired movement vector to the T1 target location despite changes in the observed amplitude of saccades due to adaptation.

\section{Discussion}

Using the McLaughlin (1967) task, after fixation of a central visual target (T0) a saccade target was presented (T1). Upon saccade initiation this target was turned off and another target (T2) closer to (backward adaptation) or further from (forward adaptation) T0 was illuminated. The introduction of this visual error by moving surreptitiously the visual target during the primary saccade led to systematic reductions or increases in saccade amplitudes. Adaptive changes in saccade amplitudes persisted even when the second target was not illuminated (probe trials) and no visual feedback was provided to the subject. Predictions of two alternative hypotheses about the role of the superior colliculus in mediation of this change in motor output (despite identical sensory inputs) were tested. As described above, these alternatives make differential predictions concerning the locus of motorrelated activity in the SC map of saccade vector. Data from 37 SC neurons in the deeper layers were recorded before and during backward adaptation. Thirteen neurons were recorded during forward adaptation. All data in our sample were consistent with the hypothesis that the locus of SC activity remained unchanged during adaptation despite changes in saccade amplitude. Data were inconsistent with the hypothesis that the locus of SC activity is remapped during adaptation. This is a strong indication that the motor command produced by the SC is not specifying the amplitude and direction of the observed saccade during shortterm adaptation produced using the McLaughlin task. Instead the SC motor command appears to specify a desired movement to the location of the T1 target. Some additional signal must reshape or bypass the collicular output to alter the amplitudes of observed movements.

Based on differences in the kinematics of saccades during backward ("gain-down") but not during forward ("gain-up") adaptation Ethier and colleagues (Ethier et al., 2008) suggest that different neural mechanisms produce the observed changes in saccade amplitude. These authors note that during backward adaptation, saccade velocities are reduced and durations slightly increased when compared with amplitude- and directionmatched movements made before adaptation (note that this is not a consistent finding across adaptation studies (for comparison, see Fitzgibbon et al., 1986; Straube and Deubel, 1995; Frens and Van Opstal, 1997; Alahyane and Pélisson, 2005). Ethier and colleagues suggest that reduction in adapted saccade velocity could indicate a change in the dynamic controller (saccadic burst generator) that is hypothesized to specify saccade kinematics. During forward adaptation they see no changes in saccade kinematics and suggest that amplitude changes in this case are the result of remapping: a change in the specification of the desired saccade vector. Our results indicating no change in SC activity during backward or forward adaptation are inconsistent with the hypothesis that the SC motor command is altered during either forward or backward adaptation.

Frens and Van Opstal (1997) reported that a subset (11/30) of neurons recorded in the deeper SC layers had activity that was unaltered during adaptation. In part due to small changes in movement amplitude during adaptation and the variability in saccade size during and after adaptation, results for the other 
reported neurons were ambiguous. It is less clear whether our data coincide with the results of another report of the role of the SC in saccadic adaptation (Takeichi et al., 2007). In this report, two measures of neural activity were used to assess changes in the SC command during adaptation. In one measure the number of action potentials in the saccade-related burst (defined as action potentials that occurred when discharge rates were $>40 \mathrm{sp} / \mathrm{s}$ ) was compared before and after both forward and backward adaptation. Due primarily to an increase in the number of spikes before movement onset during backward adaptation these authors report that SC activity is altered during adaptation. This measure of neural activity in the SC may not bear directly on the issue at hand: whether or not the SC is specifying the vector of the observed saccade or the vector of the planned saccade to the T1 location. The number of spikes in the motor-related burst could change without altering the location of the active population within the map of saccade vector. If this were the case, the increased or decreased number of spikes would not necessarily contribute to an altered movement vector and no conclusions about whether the T1 location is remapped in the SC or not would be possible. In a second measure of SC activity, Takeichi and colleagues analyzed movement fields before and after saccadic adaptation. During backward adaptation $\sim 50 \%$ of their neurons had altered movement fields after adaptation. Note that in some examples the portion of the movement field that was altered after adaptation was not related to movements to the T1 location nor to the amplitude and direction of the postadaptation saccades to T1. Nonetheless the authors interpret their altered movement fields as evidence that the SC is encoding the observed changes in saccade amplitude. However, as shown above, dramatic differences in movement fields before and after adaptation are predicted by the hypothesis that the SC does not specify the vector of the observed (postadaptation) saccade. Rather, altered movement fields (for example, Figs. 5-7) are consistent with the notion that despite large changes in saccade amplitude during adaptation, the locus of motor activity within the SC map of saccade vector does not change systematically. The data presented here indicate that the SC continues to request a saccade to the $\mathrm{T} 1$ target location and that adaptation-induced changes in saccade amplitude are a result of altered activity elsewhere in the saccade generating circuitry.

One different method also used to address the role of the SC in saccadic adaptation has been to evoke saccades by electrical stimulation. Movements evoked in this way have been reported to be
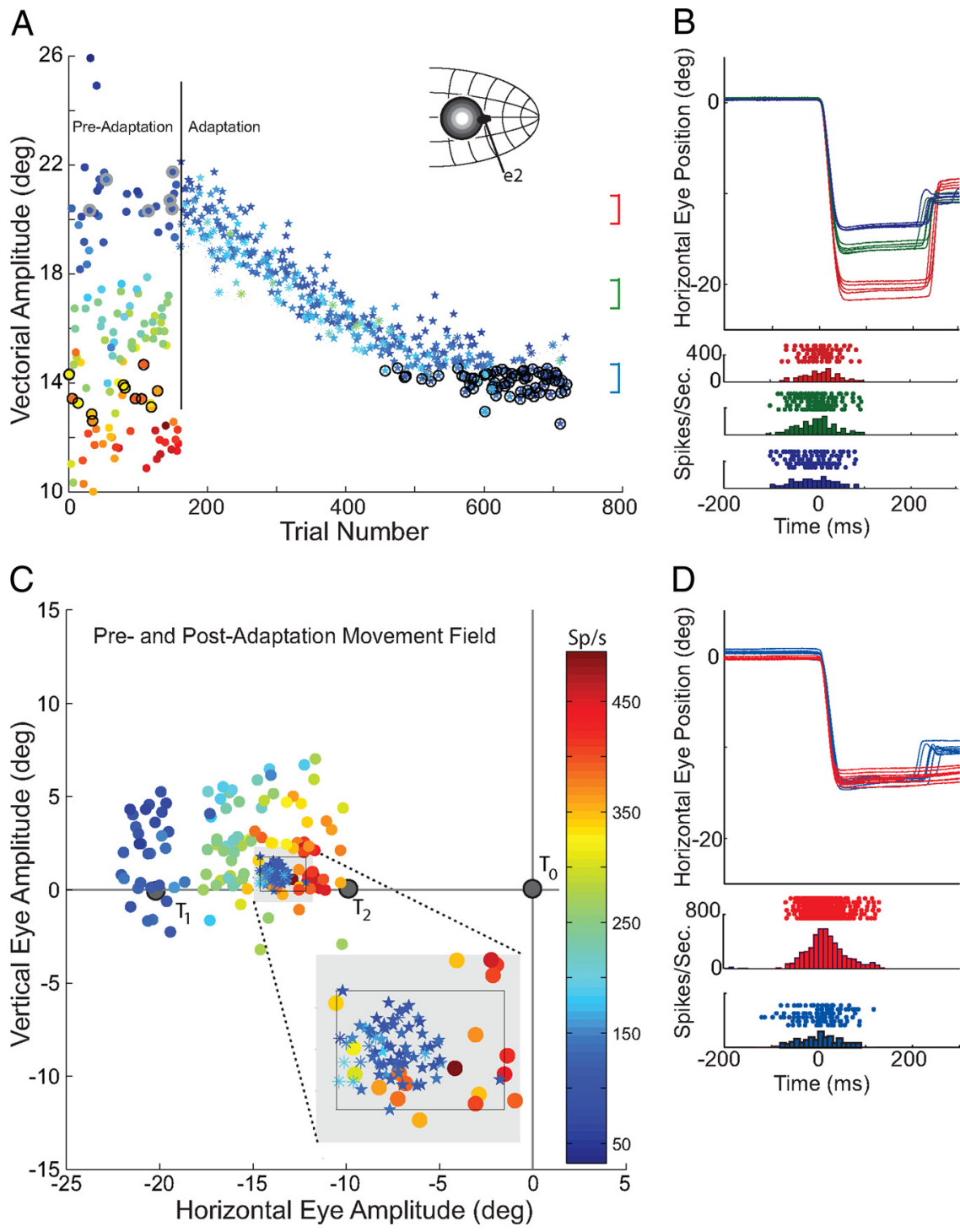

Figure 5. Neural activity before and after backward adaptation. In $\boldsymbol{A}$, the data from cell U2 plots the vectorial amplitude of saccades as a function of trial number. The first 175 trials make up the preadaptation movement field (shown in Fig. 4). Movements to the right of the vertical line are adaptation trials to $\mathrm{T} 1$ (stars) and randomly interleaved probe trials to T1 (asterisks). Also shown in $\boldsymbol{A}$ are three groups of trials that are compared in later analyses. Postadaptation trials to $\mathrm{T} 1$ (encircled in black) are compared with preadaptation movements to $\mathrm{T} 1$ (points encircled in gray), and are also compared with preadaptation movements having matched amplitudes and directions (encircled in black; also shown within the black box in $\boldsymbol{C}$ ). Inset in $\boldsymbol{A}$ shows a schematic of the SC and the hypothetical location of the active population during preadaptation trials to $\mathrm{T} 1$ and the location of the recorded neuron. Six individual trials were selected from 3 groups of movements: adaptation trials at the start (red), in the middle(green) and at the end (blue) of adaptation. These movements are superimposed in $\boldsymbol{B}$ and rasters and histograms are shown aligned on the start of saccades. In $C$, the preadaptation movement field is replotted with T0, T1 and T2 locations indicated. Superimposed on this plot are postadaptation trials (those encircled in black in A) to $\mathrm{T} 1$ (and this region is expanded in the inset). $\boldsymbol{D}$, Two sets of individual trials were randomly selected for display from within the movement field region indicated by the box in the inset; before adaptation are shown in red, after adaptation in blue. Color bar in $\boldsymbol{C}$ applies to data in both $\boldsymbol{A}$ and $\boldsymbol{C}$.

unaltered by saccadic adaptation (Fitzgibbon et al., 1986; Melis and van Gisbergen, 1996) suggesting that observed changes in saccade amplitude are not the result of an additional signal that is universally applied to the collicular output via some downstream change. This result appears to be in conflict with the results illustrated above. However, the interpretation of the electrical stimulation data depends on the assumption that stimulation of the SC recruits the identical network of neurons that is involved in producing the adapted movements. This is a difficult assumption to evaluate, and of course in its most rigorous incarnation is clearly false since visual pathways are not activated as part of a visuo- 

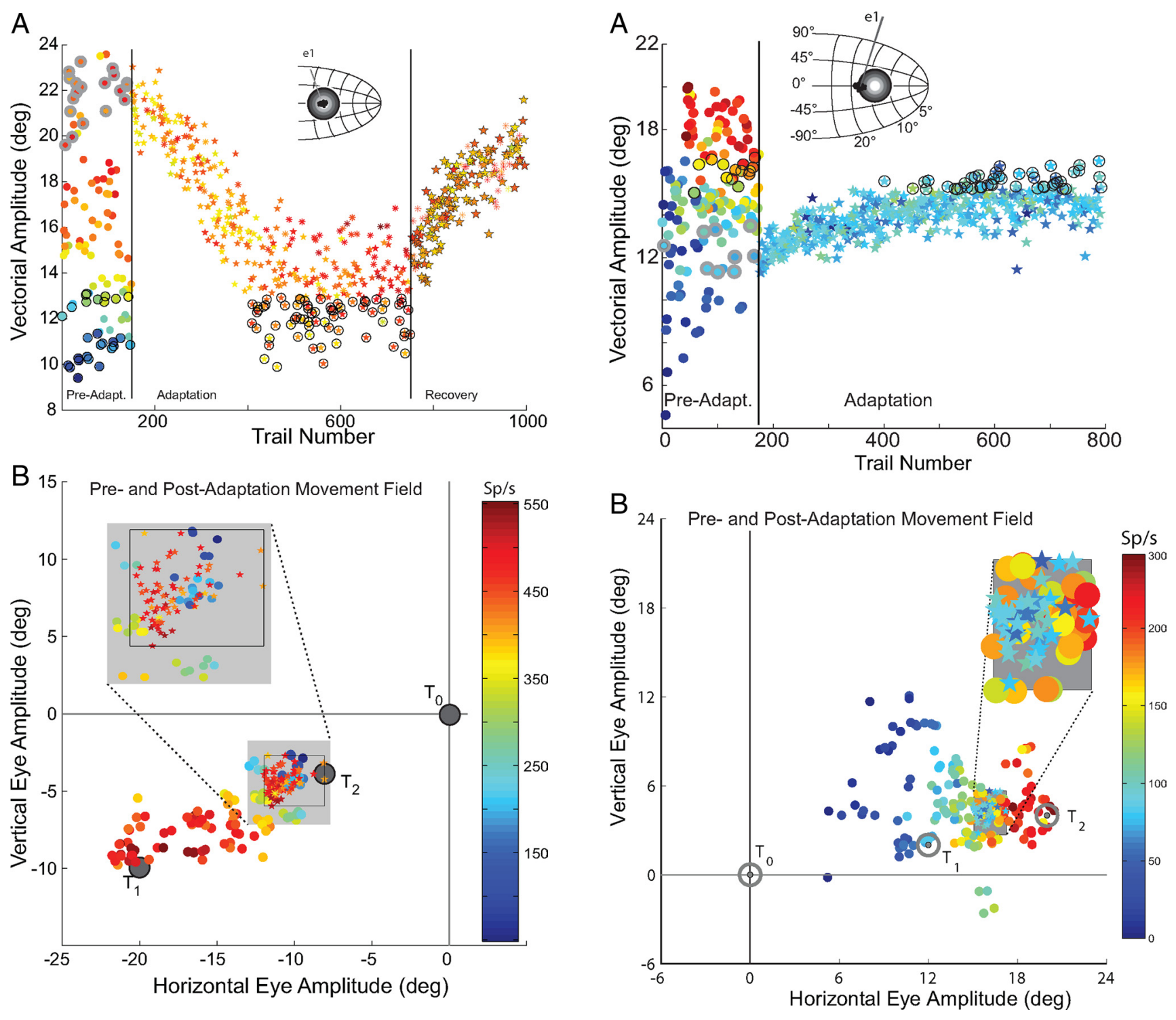

Figure 6. Example with electrode position e2. This figure has the same layout as Figure 5 but demonstrates the results of placing the $T 1$ target during preadaptation such that the recorded neuron is located near the center of the active population (inset in $\boldsymbol{A}$ ). Also shown are trials in which the T1 target is re-illuminated (recovery). Pre- and postadaptation movement fields are superimposed in B. Color bar in $\boldsymbol{B}$ applies to both $\boldsymbol{A}$ and $\boldsymbol{B}$. Recorded cell is number U12 in Table 1.

motor behavior during collicular electrical stimulation. Also, if the SC-NRTP-cerebellum-PPRF circuit is a critical loop in affecting adaptation of visual-motor behaviors, it may be that SC stimulation is failing to involve these circuits appropriately during adaptation. Additionally, using slightly different methods than those cited above, the opposite result of electrical stimulation during adaptation has also been reported (Edelman and Goldberg, 2002). In this case electrically evoked saccades were altered after saccade adaptation, suggesting that identical collicular output is altered by changes to downstream structures during the adaptive process. The data presented in the present report indicate that the SC does not alter its activity during saccade adaptation and that the observed changes either bypass the SC or are implemented downstream from the colliculus. The precise nature and location of these adaptive changes requires further study.

The data presented here are not the only examples of circumstances in which the locus of superior colliculus activity can be dissociated from the metrics of observed movements. During

Figure 7. Neural activity before and after forward adaptation. Layout is like that in Figures 5 and 6. Recorded cell is number 03 in Table 2.

saccades to remember target locations there is a systematic upward shift in movement directions. Activity in the SC, however, apparently encodes the amplitude and direction of the desired movement and the additional upward component of observed movements is added below the level of the SC (Stanford and Sparks, 1994).

The cerebellum has been implicated as a key element in saccadic adaptation (Ritchie, 1976; Optican and Robinson, 1980; Robinson and Fuchs, 2001; Robinson et al., 2002; Scudder and McGee, 2003). Cerebellar lobes VI and VII and the caudal fastigial nucleus $(\mathrm{cFN})$ receive direct and indirect inputs from a variety of saccade related structures (for review, see Voogd and Barmack, 2006). In turn, the cFN projects to brainstem regions involved in saccadic control. One route from the SC to cerebellum is via the nucleus reticularis tegmenti pontis (NRTP), and neural activity in NRTP has been reported to change during saccadic adaptation (Takeichi et al., 2005). The cFN also projects directly back to the NRTP; NRTP also receives input from a variety of cortical and subcortical structures including the frontal and supplementary eye fields and parietal cortex (for review, see 
Thier and Möck, 2006). Anatomically NRTP is well placed to integrate sensory information related to target positions and affect the execution of saccades via the cerebellum and brainstem burst generator.

During adaptation an increase in discharge before the movement starts has been reported to occur in the SC (Takeichi et al., 2007), in NRTP (Takeichi et al., 2005) and in the caudal fastigial nucleus (Inaba et al., 2003; Scudder and McGee, 2003). The mechanism for altering saccade vectors as a result of premovement activity, particularly in the SC is not clear as the locus of SC activity does not appear to change during adaptation. In contrast, an increase in premovement activity could result from repeated presentation of a single saccade target. In most saccade adaptation studies, once adaptation begins, only trials using $\mathrm{T} 1$ as the primary saccade target are presented. After several hundred presentations of the same target, without randomly interleaving trials to other locations, one might expect anticipatory activity in (for example) the SC. Increased early activity in SC, NRTP, and cFN might be a result of the target presentation scheme rather than an important element in producing the adaptive changes in saccade amplitude. The neural mechanisms that result in altered saccade metrics and the roles of NRTP, cFN and other saccaderelated structures remain to be determined. The data presented above, however, are inconsistent with remapping of the saccade vector at the level of the superior colliculus.

\section{Summary}

We tested the predictions of two alternative hypotheses about the role of the superior colliculus in mediating changes in saccade amplitude during adaptation. To test these predictions required an assessment of the locus of SC motor-related activity before, during and after adaptive changes in saccade amplitude. We found no evidence that saccade vectors are remapped at the level of the SC during either backward or forward adaptation; data were consistent with the hypothesis that the locus of SC activity remained unchanged despite changes in saccade amplitudes. We conclude that the observed amplitude changes must occur as a result of activity in other regions: NRTP-cerebellum-reticular formation network seems a likely route, but the role of higher saccade centers (FEF, SEF, parietal cortex) cannot be discounted.

\section{References}

Alahyane N, Pélisson D (2004) Eye position specificity of saccadic adaptation. Invest Ophthalmol Vis Sci 45:123-130.

Alahyane N, Pélisson D (2005) Long-lasting modifications of saccadic eye movements following adaptation induced in the double-step target paradigm. Learn Mem 12:433-443.

Cecala AL, Freedman EG (2008) Amplitude changes in response to target displacements during human eye-head movements. Vision Res 48: $149-166$.

Cecala AL, Freedman EG (2009) Head-unrestrained gaze adaptation in the rhesus macaque. J Neurophysiol 101:164-183.

Collewijn H (1977) Eye- and head movements in freely moving rabbits. J Physiol 266:471-498.

Deubel H, Wolf W, Hauske G (1986) Adaptive gain control of saccadic eye movements. Hum Neurobiol 5:245-253.

Edelman JA, Goldberg ME (2002) Effect of short-term saccadic adaptation on saccades evoked by electrical stimulation in the primate superior colliculus. J Neurophysiol 87:1915-1923.

Ethier V, Zee DS, Shadmehr R (2008) Changes in control of saccades during gain adaptation. J Neurosci 28:13929-13937.

Fitzgibbon E, Goldberg M, Segraves M (1986) Short term adaptation in the monkey. In: Adaptive processes in visual and oculomotor systems (Keller EL, Zee DS, eds), pp 329-339. Oxford: Pergamon.

Frens MA, van Opstal AJ (1994) Transfer of short-term adaptation in human saccadic eye movements. Exp Brain Res 100:293-306.
Frens MA, Van Opstal AJ (1997) Monkey superior colliculus activity during short-term saccadic adaptation. Brain Res Bull 43:473-483.

Hopp JJ, Fuchs AF (2002) Investigating the site of human saccadic adaptation with express and targeting saccades. Exp Brain Res 144:538-548.

Hopp JJ, Fuchs AF (2004) The characteristics and neuronal substrate of saccadic eye movement plasticity. Prog Neurobiol 72:27-53.

Hopp JJ, Fuchs AF (2006) Amplitude adaptation occurs where a saccade is represented as a vector and not as its components. Vision Res 46:3121-3128.

Inaba N, Iwamoto Y, Yoshida K (2003) Changes in cerebellar fastigial burst activity related to saccadic gain adaptation in the monkey. Neurosci Res 46:359-368.

Judge SJ, Richmond BJ, Chu FC (1980) Implantation of magnetic search coils for measurement of eye position: and improved method. Vision Res 20: 535-538.

McLaughlin SC (1967) Parametric adjustment in saccadic eye movements. Percept Psychophysiol 2:359-362.

Melis BJ, van Gisbergen JAM (1996) Short-term adaptation of electrically induced saccades in monkey superior colliculus. J Neurophysiol 76:1744-1758.

Miller JM, Anstis T, Templeton WB (1981) Saccadic plasticity: parametric adaptive control of retinal feedback. J Exp Psychol Hum Percept Perform 7:356-366.

Noto CT, Watanabe S, Fuchs AF (1999) Characteristics of simian adaptation fields produced by behavioral changes in saccade size and direction. J Neurophysiol 81:2798-2813.

Optican LM, Robinson DA (1980) Cerebellar-dependent adaptive control of primate saccadic system. J Neurophysiol 44:1058-1076.

Phillips JO, Fuchs AF, Ling L, Iwamoto Y, Votaw S (1997) Gain adaptation of eye and head movement components of simian gaze shifts. J Neurophysiol 78:2817-2821.

Ritchie L (1976) Effects of cerebellar lesions on saccadic eye movements. J Neurophysiol 39:1246-1256.

Robinson FR, Fuchs AF (2001) The role of the cerebellum in voluntary eye movements. Annu Rev Neurosci 24:981-1004.

Robinson FR, Fuchs AF, Noto CT (2002) Cerebellar influences on saccade plasticity. Ann N Y Acad Sci 956:155-163.

Robinson FR, Noto CT, Bevans SE (2003) Effect of visual error size on saccade adaptation in monkey. J Neurophysiol 90:1235-1244.

Scudder CA, McGee DM (2003) Adaptive modification of saccade size produces correlated changes in the discharges of fastigial nucleus neurons. J Neurophysiol 90:1011-1026.

Scudder CA, Batourina EY, Tunder GS (1998) Comparison of two methods of producing adaptation of saccade size and implications for the site of plasticity. J Neurophysiol 79:704-715.

Sparks DL, Hartwich-Young R (1989) The deep layers of the superior colliculus. Rev Oculomot Res 3:213-255.

Sparks DL, Mays LE (1981) The role of the monkey superior colliculus in the control of saccadic eye movements: a current perspective. In: Progress in oculomotor research (Fuchs A, Becker W, eds) pp 137-144. New York: Elsevier.

Stanford TR, Sparks DL (1994) Systemic errors for saccades to remembered targets: evidence for a dissociation between saccade metrics and activity in the superior colliculus. Vision Res 34:93-106.

Straube A, Deubel H (1995) Rapid gain adaptation affects the dynamics of saccadic eye movements in humans. Vision Res 35:3451-3458.

Straube A, Fuchs AF, Usher S, Robinson FR (1997) Characteristics of saccadic gain adaptation in rhesus macaques. J Neurophysiol 77:874-895.

Takeichi N, Kaneko CR, Fuchs AF (2005) Discharge of monkey nucleus reticularis tegmenti pontis neurons changes during saccade adaptation. J Neurophysiol 94:1938-1951.

Takeichi N, Kaneko CR, Fuchs AF (2007) Activity changes in monkey superior colliculus during saccade adaptation. J Neurophysiol 97:4096-4107.

Thier P, Möck M (2006) The oculomotor role of the pontine nuclei and the nucleus reticularis tegmenti pontis. Prog Brain Res 151:293-320.

Voogd J, Barmack NH (2006) Oculomotor cerebellum. Prog Brain Res 151:231-268.

Wallman J, Fuchs AF (1998) Saccadic gain modification: visual error drives motor adaptation. J Neurophysiol 80:2405-2416.

Wurtz RH, Goldberg ME (1971) Superior colliculus cell responses related to eye movements in awake monkeys. Science 171:82-84. 